\title{
Modelling electrospinning of nanofibres
}

\author{
Tomasz A. Kowalewski ${ }^{1, *}$ and Serge Barral ${ }^{1}$ \\ ${ }^{1}$ IPPT PAN, Polish Academy of Sciences, Warsaw
}

\begin{abstract}
Electrospinning is based on so-called bending instability which results in an erratic spiralling motion of the liquid jet as it proceeds towards a collecting electrode, where it is eventually deposited as a mat of micro/nanosized fibres. Most electrospinning models formulated within the slender approximation rely, however, on an inconsistent description of electrostatic interactions which renders them grossly inappropriate whenever the discretization is either too coarse or too fine. The present work aims at proposing a discrete slender model which is numerically consistent (allowing use of arbitrary fine meshes) and remains accurate even for coarse meshes. At the same time, efficient numerical techniques based on hierarchical charge clustering are introduced that drastically decrease computational times. Finally, a versatile boundary value method is implemented to enforce fixed-potential boundary conditions, allowing realistic electrode configurations to be investigated.
\end{abstract}

Copyright line will be provided by the publisher

Electrospinning is a simple and relatively inexpensive mean of producing continuous fibres with diameters ranging from micrometers down to a few nanometres. Nonwovens of electrospun fibres are obtained from a jet of polymer solution stretched by an electric field. Although most polymeric solutions or melts may be used for electrospinning, the achievement of stable operation is usually the result of a tedious trial-and-error parametrical optimization procedure. On the other hand the physical and mathematical description of the electrospinning process remain in a premature state. The existing discrete models are based on point charges connected by dumbbell elements [1-5]. One serious concern relates to the evaluation of short-range interactions, which in the case of standard discrete integration methods require very dense grids due to the large contribution of short-range electrostatic interactions within distances of the order of the fibre radius $[1,6]$. As the fibre radius is about $10^{3}-10^{5}$ times smaller than the macroscopic scales of interest, it appears most desirable to devise a discrete model that exploits the slenderness of the fibre to evaluate short-range interactions in an efficient manner. Likewise, the computation of long-range electrostatic interactions can easily become intractable due to the $\mathcal{O}\left(n^{2}\right)$ operation count for a pairwise evaluation of interactions between $n$ elements. In addition current numerical models are based on the assumption of a static external electric field, whereas in reality the external field is modulated by the net space charge of the fibre so as to keep constant the potential over the electrodes. An efficient handling of long-range interactions can theoretically achieve $\mathcal{O}(n \log n)$ operation count, or even $\mathcal{O}(n)$ for the fast multipole method (FMM). The proposed here treecode algorithm [7] considers particle-cluster interactions and achieves $\mathcal{O}(n \log n)$ complexity. Generally it is a time-dependent three-dimensional generalization of known slender models $[2,4,5,8]$, and solves the mass and momentum conservation equation for the Maxwell viscoelastic fluid. The mass conservation and momentum equations accordingly read

$$
\frac{\partial a^{2}}{\partial t}+\frac{\partial\left(v a^{2}\right)}{\partial \xi}=0, \quad \rho \pi a^{2} \ddot{\mathbf{x}}=\frac{\partial\left(\pi a^{2} \tau \hat{\mathbf{t}}+\pi \gamma a \hat{\mathbf{t}}\right)}{\partial \xi}+\lambda \mathbf{E} .
$$

Here $a$ is the fibre radius, $v$ is the velocity of the fibre, $\xi$ is the arc length along the fibre, $\rho$ is the mass density, $\ddot{\mathrm{x}}$ denotes the acceleration vector, $\gamma$ is the surface tension coefficient, $\hat{\mathbf{t}}$ is the local unit vector tangent to the fibre, $\lambda$ is the linear charge density, $\mathbf{E}$ is the electric field and $\tau$ is the viscoelastic stress. The stress is given by a Maxwell viscoelastic constitutive equation

$$
\dot{\tau}=G\left(\varepsilon-\frac{\tau}{\mu}\right), \quad \varepsilon \equiv \frac{\partial \dot{\mathbf{x}}}{\partial \xi} \cdot \hat{\mathbf{t}} .
$$

Here $G$ is the elastic modulus, $\mu$ the fluid viscosity and $\varepsilon$ the Lagrangian axial strain.

A Lagrangian discrete model is used to solve the problem numerically. The fibre is first decomposed into discrete charged elements $Q_{i+\frac{1}{2}}$, the length of which is typically much greater than the fibre radius, but smaller than other characteristic lengths of interest (such as the curvature radius). The equation of motion is then resolved at the interfaces of each element, where all forces are priorly evaluated. After displacements have been calculated, the radius in the central sections of elements $Q_{i+\frac{1}{2}}$, is straightforwardly obtained since the volume of each element is conserved. The forces at each collocation node are computed from the following discrete form of the momentum equation,

$$
\ddot{\mathbf{x}}_{i}=\frac{1}{\rho \pi a_{i}^{2}} \frac{\pi\left(a^{2} \tau\right)_{i+\frac{1}{2}}-\pi\left(a^{2} \tau\right)_{i-\frac{1}{2}}+\pi \gamma\left(a_{i+\frac{1}{2}}-a_{i-\frac{1}{2}}\right)}{\frac{1}{2}\left(\left|\mathbf{x}_{i}-\mathbf{x}_{i-1}\right|+\left|\mathbf{x}_{i+1}-\mathbf{x}_{i}\right|\right)} \hat{\mathbf{t}}_{i}+\frac{1}{\rho}\left(\tau_{i}+\frac{\gamma}{a_{i}}\right) \kappa_{i} \hat{\mathbf{n}}_{i}+\lambda_{i} \mathbf{E}_{i} .
$$

\footnotetext{
* Corresponding author: e-mail: tkowale@ippt.gov.pl, Phone: +48 22 8269803, Fax: +48 228269815
} 

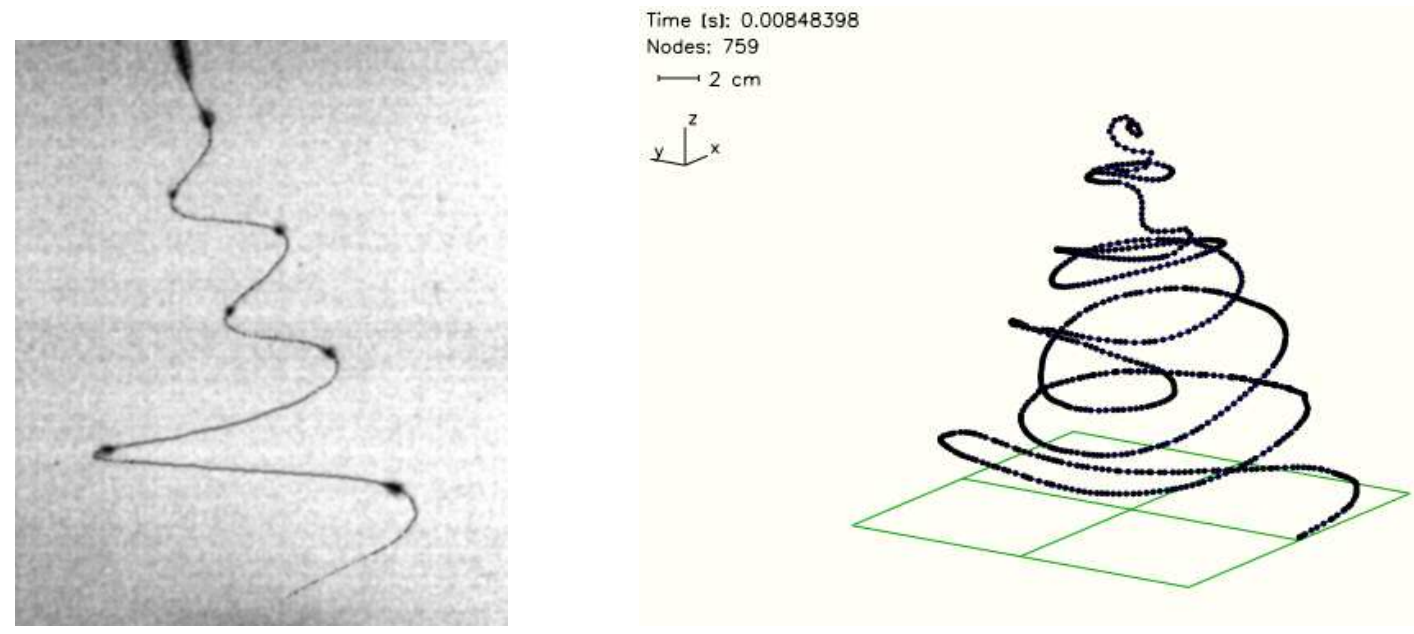

Fig. 1 Observed electrospinning nanofiber (left) and its simulated counterpart (right).

The values $a_{i}, \tau_{i}, \lambda_{i}$ required at the collocation nodes are linearly interpolated from those computed in the central section of the neighbouring elements. The local tangent vector $\hat{\mathbf{t}}_{i}$ and curvature vector $\kappa \hat{\mathbf{n}}_{i}$ are computed from the approximate osculating circle defined by $\left(N_{i-1}, N_{i}, N_{i+1}\right)$.

Time integration is realized with a classical leapfrog scheme. Dynamic refinement is used in simulations to maintain the size of elements below a prescribed characteristic length $\ell_{\max }$; whenever an element is elongated beyond $\ell_{\max }$, it is split into two elements, each containing half the charge and mass of the initial element.

The needle and the grounded collector are idealized by a point-charge/plate capacitor configuration. By implementing treecode algorithm charge elements are recursively clustered and the monopole coefficients (charge and centre of charge) of the clusters are computed. The field at a location $N_{i}$ is then computed by considering only the largest clusters which are sufficiently well separated. At each time step, a binary tree is thus constituted by recursively grouping neighbours two by two, calculating at each level the smallest enclosing spheres that contain the cluster pairs. At the inlet (tip of the needle), the volume flow rate $Q_{v}$, the surface charge density $\sigma_{0}$ and the fibre radius $a_{0}$ are prescribed. The initial stress is set to zero. A small random perturbation to the position of each element introduced at the inlet is imposed, so as to initiate the bending instability. The magnitude of this perturbation has no notable influence on the simulation results, provided that it is small enough [7].

Behaviour of the code was tested using parameters typical for the experiment. Figure 1 shows an example of high speed recording of electrospinning nanofibre of PEO (polyethylene oxide) aqueous solution and numerical simulation performed with the present code. The main futures of the bending process are well reproduced, except missing initial straight part of the jet - not included in the present model. Several test runs performed confirmed general ability of the code to replicate our previous findings, i.e. increased electrical potential, solution viscosity and elastic modulus decreased jet sweeping amplitude, effectively seen as decreasing of the spiral cone. New algorithm appeared to be robust and fast comparing with our previous attempts [1], allowing to perform several parametric studies in a relatively short time. Father development is necessary to implement development of the initial destabilisation of the straight part of the jet and variation of its physical properties during polymer solidification and solvent evaporation.

Acknowledgements This work was supported by Polish Ministry of Science grant No. N508-03131/1740.

\section{References}

[1] T. A. Kowalewski, S. Blonski, and S. Barral, Bull. Polish Acad. Sci. 53, 385 (2005).

[2] J. J. Feng, J. Non-Newtonian Fluid Mech. 116, 55 (2003).

[3] A. L. Yarin, S. Koombhongse, and D. H. Reneker, J. Appl. Phys. 89, 3018 (2001).

[4] M. M. Hohman, M. Shin, G. Rutledge, and M. P. Brenner, Phys. Fluids 13, 2201 (2001).

[5] J. J. Feng, Phys. Fluids 14, 3912 (2002).

[6] V. Khayms, Advanced Propulsion for Microsatellites, PhD thesis, Massachusetts Institute of Technology, 2000.

[7] T. Kowalewski, S. Barral, and T. Kowalczyk, in: IUTAM Symposium on Modelling Nanomaterials and Nanosystems, Aalborg, Denmark, edited by R. Pyrz and J. Rauhe (Springer, Netherlands, 2009), pp. 279-292.

[8] C. P. Carroll and Y. L. Joo, Phys. Fluids 18 (2006). 